\title{
A COX-2 far?
}

Findings from a newly published randomized study, combined with previous data regarding patient safety, should put a halt to interest in cyclooxygenase 2 (COX-2) inhibition as prostate cancer chemoprevention, according to the study authors.

COX-2 has been suggested as a target for chemoprevention, owing to its role in the development and progression of prostate cancer. The team performed a randomized controlled trial, in which 28 men with localized prostate cancer received either $400 \mathrm{mg}$ of the COX-2 inhibitor celecoxib or placebo twice daily for 4 weeks before undergoing prostatectomy. Specimens were then analysed for levels of apoptosis, prostaglandins, and the androgen receptor; serum PSA and opiate use postoperatively was also assessed.

Although celecoxib therapy was, in general, well tolerated, one patient suffered a nonfatal postoperative myocardial infarction, thought to be caused by perioperative blood loss. No significant changes were observed in serum PSA levels between baseline and postoperative assessment, or between the placebo and celecoxib arms. Likewise, apoptosis indices in tumour cells and in benign cells were not significantly different between the groups, and levels of prostaglandins and androgen receptors were also similar.

These data are in agreement with previous studies suggesting that COX-2 inhibition does not benefit men with prostate cancer, and the authors assert that their results preclude further investigation of COX-2 inhibition in chemoprevention. Lead author Tomasz Beer commented: "To the extent that taking such agents or other NSAIDs has been associated with a reduced risk of prostate cancer in some epidemiological studies, our study does not provide a biological basis for these observations."

Annette Fenner

ORIGINAL ARTICLE Flamiatos, J. F. et al. COX-2

inhibition for prostate cancer chemoprevention:

double-blind randomized study of pre-

prostatectomy celecoxib or placebo. BJU Int

http://dx.doi.org/10.1111/bju.13612 (2016) 